\title{
Hydroxychloroquine in the treatment of adult patients with Covid-19 infection in a primary care setting (LIBERTY): A structured summary of a study protocol for a randomised controlled trial
}

Petri J. Vainio ${ }^{1,2}$, Pauliina Hietasalo ${ }^{3}$, Anna-Liisa Koivisto ${ }^{3}$, Susanna Kääriäinen ${ }^{4}$, Jari Turunen ${ }^{5}$, Mika Virtala $^{3}$, Jouni Vuorinen ${ }^{5}$ and Mika Scheinin ${ }^{1,2,4^{*}}$ (D)

\begin{abstract}
Objectives: The primary objective of this study is to evaluate the therapeutic potential of hydroxychloroquine (HCQ) in the treatment of adult patients with PCR-confirmed Covid-19 infection in a primary open-care setting, as compared to placebo. The study hypothesis is that treatment with HCQ will reduce the risk of hospitalization because of Covid-19 infection, and the sample size estimate of the study is based on the need to test this hypothesis.

The secondary objectives of the study are:

- to evaluate the safety and tolerability of HCQ in the treatment of adult patients with PCR-confirmed Covid-19 infection in a primary open-care setting, as compared to placebo;

- to collect experience of the use of HCQ in the treatment of Covid-19 infection in outpatients, in order to be able to identify patient characteristics that predict specific treatment responses (favourable or unfavourable); this objective will also be addressed by post-hoc subgroup analysis of the study results and by meta-analysis of pooled patient data from other clinical trials of HCQ in outpatients; and

- to evaluate the impact of Covid-19 infection and its treatment on the mental health and well-being of the study participants.
\end{abstract}

In addition, if the data allow, the study has the following exploratory objectives:

- to evaluate the extent and duration of SARS-CoV-2 viral shedding by PCR testing of nasopharyngeal swab samples in study subjects treated with $\mathrm{HCQ}$, as compared to placebo;

(Continued on next page)

\footnotetext{
* Correspondence: mschein@utu.fi

${ }^{1}$ Institute of Biomedicine, University of Turku, Turku, Finland

${ }^{2}$ Unit of Clinical Pharmacology, Turku University Hospital, Turku, Finland

Full list of author information is available at the end of the article
}

C C The Author(s). 2021 Open Access This article is licensed under a Creative Commons Attribution 4.0 International License, which permits use, sharing, adaptation, distribution and reproduction in any medium or format, as long as you give appropriate credit to the original author(s) and the source, provide a link to the Creative Commons licence, and indicate if changes were made. The images or other third party material in this article are included in the article's Creative Commons licence, unless indicated otherwise in a credit line to the material. If material is not included in the article's Creative Commons licence and your intended use is not permitted by statutory regulation or exceeds the permitted use, you will need to obtain permission directly from the copyright holder. To view a copy of this licence, visit http://creativecommons.org/licenses/by/4.0/ The Creative Commons Public Domain Dedication waiver (http://creativecommons.org/publicdomain/zero/1.0/) applies to the data made available in this article, unless otherwise stated in a credit line to the data. 
(Continued from previous page)

- to evaluate the extent and time course of SARS-CoV-2 virus-specific antibody responses in serum of study subjects treated with $\mathrm{HCQ}$, as compared to placebo;

- to evaluate other possible biomarker changes in blood in study subjects treated with HCQ, as compared to placebo;

- to explore the possible effects of genetic variation in drug metabolizing enzymes on HCQ-related outcomes in the study population;

- to explore the associations of HCQ-related outcome variables with other patient characteristics, e.g. HLA haplotypes, HCQ concentrations, demographic variables, disease history and concomitant medications.

Trial design: This is a phase 2, placebo-controlled, double-blind, randomized, parallel-group treatment trial comparing HCQ with placebo in outpatients with Covid-19 infection. Participants will be randomized in a 1:1 ratio to the two treatment arms.

\section{Participants:}

Main inclusion criteria:

1. Males and females $>40$ years of age, or $18-40$ years of age with one or both of the following: i. diabetes mellitus (type 1 or type 2); ii. BMI > $35 \mathrm{~kg} / \mathrm{m}^{2}$;

2. Valid independent informed consent obtained;

3. Symptoms typical of Covid-19 infection, according to criteria specified in the study protocol. The onset of symptoms must be within 5 days of enrolment;

4. Positive SARS-CoV-2 PCR test result of a nasopharyngeal swab sample.

Main exclusion criteria:

1. Suspected severe or moderately severe pneumonia, presenting with any of the following: respiratory rate $>26$ breaths/min; significant respiratory distress; or $\mathrm{SpO}_{2} \leq 94 \%$ on room air;

2. Requiring treatment in the hospital, according to the treating physician's judgement;

3. Any contraindication to treatment with $\mathrm{HCQ}$;

4. Pregnancy or lactation.

The trial will be conducted at seven study sites in a primary public health care setting in the region of Satakunta, Finland.

Intervention and comparator: Participants will be randomized to receive either HCQ capsules at 300 mg twice a day for one day and then 200 mg twice a day for 6 days, or placebo capsules for 7 days.

Main outcomes: The primary endpoint of the study is the number of hospitalizations due to Covid-19 infection within four weeks of entry into the study.

The secondary endpoints of the study include the following:

- duration and severity of Covid-19-related symptoms, as reported by daily self-assessments;

- number of Intensive Care Unit treatment episodes due to Covid-19 infection within four weeks of entry into the study;

- number of deaths due to Covid-19 infection within four weeks of entry into the study;

- number of treatment-related adverse events (AEs) and serious AEs (SAEs);

- all-cause hospitalizations and mortality within six months of entry into the study; and

- self-assessed symptoms of anxiety, as assessed with repeated administration of the Generalized Anxiety Disorder 7-item scale (GAD-7).

The exploratory endpoints of the study include the following:

- extent and duration of SARS-CoV-2 viral shedding and virus-specific antibody responses in serum; and

- possible other blood biomarker changes.

(Continued on next page) 


\section{(Continued from previous page)}

Randomisation: Eligible study participants are randomly allocated into two treatment arms (1:1 ratio). The randomization list has been generated using Viedoc ${ }^{\text {TM }}$ (Viedoc Technologies AB, Uppsala, Sweden) that is used as an electronic data capture system for this study.

Blinding (masking): The participants and all study personnel remain blinded to the treatment allocation by having both IMPs packed in identical containers. Masking of the treatments was performed by re-formulation of the IMPs so that the HCQ capsules and the placebo capsules have identical appearance.

Numbers to be randomised (sample size): 600 participants are to be randomised with 300 in each arm.

Trial Status: Protocol version 2, dated 14 July 2020; recruitment is expected to start in December, 2020, and to be completed in June, 2021.

Trial registration: EudraCT 2020-002038-33, registered 26 June 2020

Full protocol: The full protocol is attached as an additional file, accessible from the Trials website (Additional file 1). The protocol has been redacted to conform with privacy regulations by deleting the names and contact information of individuals mentioned in the protocol but not listed as authors in this communication. In the interest of expediting dissemination of this material, the familiar formatting has been eliminated; this Letter serves as a summary of the key elements of the full protocol.

Keywords: COVID-19, Randomised controlled trial, protocol, hydroxychloroquine, primary care, open care, public health care, hospitalization

\section{Supplementary Information}

The online version contains supplementary material available at https://doi. org/10.1186/s13063-020-04989-6.

Additional file 1. Full Study Protocol.

\section{Acknowledgements}

Not applicable

\section{Authors' contributions}

PJV: principal investigator, protocol design, manuscript preparation; PH: study logistics, manuscript preparation; SK: protocol design, medical writing, regulatory affairs, manuscript preparation; A-LK: protocol design, study logistics, manuscript preparation; MS: study conception, study design, medical writing, manuscript preparation; JT: protocol design, data management; MV: study conception, study design, study logistics, manuscript preparation; JV: protocol design, statistical analysis. The authors read and approved the final manuscript.

\section{Authors' information}

Not applicable.

\section{Funding}

This research is funded by the participating municipalities, with the City of Pori as responsible sponsor. The study has received donations (so far, 32.000 euro) from Kuusisto Group, Osuuskauppa Keula and three other private enterprises operating in the region of Satakunta. None of these enterprises are involved in health care or pharmaceuticals, and they have no role in the design of the study or the collection, analysis, and interpretation of data and in writing the manuscript. Additional funding is being sought in the form of grants and donations.

\section{Availability of data and materials}

The study data will be available from the authors on reasonable request for meta-analysis and other purposes covered by the participants' consent (contact information for requests: mschein@utu.fi).

\section{Ethics approval and consent to participate}

Conditional approval of protocol version 1 was received on 10 July 2020 from Finland's National Committee on Medical Research Ethics, TUKIJA (Dnro
88/06.00.01/2020). Final approval of protocol version 2 and the revised ICF was received from the same ethics committee on 17 August 2020. The authors certify that this trial has received ethical approval from the appropriate ethical committee as described above. Potential study subjects will first receive written and verbal information on the study and will have an opportunity to ask questions. If a subject decides to participate in the trial, written informed consent will be obtained before any study-related procedures are performed. A copy of the signed ICF will be provided to the subject.

\section{Consent for publication}

Not applicable

\section{Competing interests}

SK and MS are employed by Clinical Research Services Turku - CRST Oy, a contract research organization engaged in commercial clinical trials for the pharmaceutical industry. All authors declare that they have no competing interests related to this trial.

\section{Author details}

${ }^{1}$ Institute of Biomedicine, University of Turku, Turku, Finland. ${ }^{2}$ Unit of Clinical Pharmacology, Turku University Hospital, Turku, Finland. ${ }^{3}$ Porin perusturva, Pori, Finland. ${ }^{4}$ Clinical Research Services Turku - CRST Oy, Turku, Finland. ${ }^{5}$ Oy 4Pharma Ltd., Turku, Finland.

Received: 15 December 2020 Accepted: 18 December 2020

Published online: 11 January 2021

\section{Publisher's Note}

Springer Nature remains neutral with regard to jurisdictional claims in published maps and institutional affiliations. 